\title{
A New Type of Chebyshev Quadrature*
}

\author{
By R. E. Barnhill, J. E. Dennis, Jr. and G. M. Nielson
}

Abstract. A Chebyshev quadrature is of the form

$$
\int_{-1}^{1} w(x) f(x) d x \simeq c \sum_{k=1}^{n} f\left(x_{k}\right) .
$$

It is usually desirable that the nodes $x_{k}$ be in the interval of integration and that the quadrature be exact for as many monomials as possible (i.e., the first $n+1$ monomials). For $n=1, \cdots, 7$ and 9 , such a choice of nodes is possible, but for $n=8$ and $n>9$, the nodes are complex. In this note, the idea used is that the $l^{2}$-norm of the deviations of the first $n+1$ monomials from their moments be a minimum. Numerical calculations are carried out for $n=8,10$, and 11 and one interesting feature of the numerical results is that a "multiple" node at the origin is required. The above idea is then generalized to a minimization of the $l^{2}$-norm of the deviations of the first $k$ monomials, $k \geqq n+1$, including $k=\infty$, and corresponding numerical results are presented.

1. Introduction. A Chebyshev quadrature is of the form

$$
\int_{-1}^{1} w(x) f(x) d x \simeq c \sum_{k=1}^{n} f\left(x_{k}\right) .
$$

There are several advantages in having a constant weight $c$, one of which is the following [2, p. 71]: Suppose that numerical errors are made in evaluating the point functionals $f\left(x_{k}\right)$ and that these errors are normally distributed with a common variance. If a quadrature of the form

$$
\int_{-1}^{1} w(x) f(x) d x \simeq \sum_{k=1}^{n} A_{k} f\left(x_{k}\right)
$$

is exact for the function 1, i.e.,

$$
\int_{-1}^{1} w(x) d x=\sum_{k=1}^{n} A_{k},
$$

then the standard deviation of the error in the quadrature sum due to the above cause is minimized by taking all the $A_{k}$ to be equal, i.e.,

$$
A_{k}=\frac{1}{n} \int_{-1}^{1} w(x) d x, \quad k=1, \cdots, n .
$$

It is desirable for most purposes that the quadrature nodes $x_{k}$ be in the interval

Received July 11, 1968.

* This work was supported by the National Science Foundation under Grant GP 5906 to The University of Utah. The computations were done on the Univac 1108 at the University of Utah Computer Center. 
$[-1,1]$. Since there are $n$ nodes and one weight, any $n+1$ functions that form a Chebyshev set can be integrated exactly. Conventionally, these are taken to be the first $n+1$ monomials $1, x, \cdots, x^{n}$. For $n=1, \cdots, 7$, and 9 , there exist Chebyshev quadratures with nodes in $[-1,1]$ that integrate the first $n+1$ monomials exactly. For $n=8$ and $n>9$, the first $n+1$ monomials can be integrated exactly, but some of the nodes are complex.

With this as background, there are various alternatives that can be pursued. One of these is to maintain the requirement of a constant weight and the nodes being in $[-1,1]$ and to find the highest algebraic degree of precision $m=m(n)$. This possibility has been explored and references to its literature are given in the paper by Meir and Sharma [3].

A different approach is used in Section 2 of this paper. We consider $w(x) \equiv 1$, $c=2 / n$, and the nodes constrained to be in $[-1,1]$. We formulate the problem as follows: find a quadrature of the form

$$
\frac{2}{n} \sum_{k=1}^{n} f\left(x_{k}\right)
$$

so that

$$
\sum_{j=0}^{n}\left[\frac{2}{n} \sum_{k=1}^{n} x_{k}{ }^{j}-m_{j}\right]^{2}
$$

is a minimum, where $m_{j}=\int_{-1}^{1} x^{j} d x$ is the $j$ th moment. That is, we want to minimize the $l^{2}$-norm of the "deviations" of the first $n+1$ monomials from their moments.

2. Three New Quadratures. This problem has been solved numerically for the cases $n=8,10$, and 11 . The solutions for these cases were found by the application of a generalized Newton's method. Under certain conditions, the Newton iterates converge to a minimum of (2). These conditions are given in the article by BenIsrael [1]. The computer programs were checked by calculating the classical weights and nodes for $n=6,7$ and 9 . Symmetry was assumed for all cases** and so only nonnegative values need be considered. The cases $n=8,10$, and 11 have the interesting common property that if only positive nodes were used in the initial approximations, then convergence was not obtained either with the generalized Newton's method or with the gradient methods used. That is, for $n=8$ and 10, a double node at zero and, for $n=11$, a triple node at zero, were required for convergence.

For $n=8$, the results are the following:

(The nodes are labelled from left to right in $[-1,1]$.)

$$
\begin{array}{ll}
-x_{1}=x_{8}=0.90044 & 55323, \\
-x_{2}=x_{7}=0.55898 & 89280, \\
-x_{3}=x_{6}=0.45850 & 78272, \\
-x_{4}=x_{5}=0.0 . &
\end{array}
$$

\footnotetext{
** For $n=8$, the same results were unchanged (to at least seven decimals) if symmetry was not assumed and, also, if $c$ was not assumed to be $2 / n$.
} 
The minimum of (2) for $n=8$ is $0.79221 \times 10^{-6}$ and the corresponding approximations to the moments are the following:

$$
\begin{aligned}
& \frac{1}{4} \sum_{i=1}^{8} x_{i}{ }^{2}=0.66675 \text { vs. } 2 / 3, \\
& \frac{1}{4} \sum_{i=1}^{8} x_{i}{ }^{4}=0.39962 \text { vs. } 2 / 5, \\
& \frac{1}{4} \sum_{i=1}^{8} x_{i}{ }^{6}=0.28641 \text { vs. } 2 / 7, \\
& \frac{1}{4} \sum_{i=1}^{8} x_{i}{ }^{8}=0.22183 \text { vs. } 2 / 9 .
\end{aligned}
$$

These results were found after four iterations of the generalized Newton's method, with the initial approximation $(.9015, .568, .468,0.0)$. The calculation was terminated when there was no change in the first twelve digits in the nodes. This initial approximation was found by an interpolation at $n=8$ on the graph of $n$ vs. the classical Chebyshev nodes, with the alteration that 0.0 was included, according to the above remarks. The initial approximations for $n=10$ and 11 were found by an extrapolation from the same graph.

The numerical results for $n=10$ and 11 are the following:

$$
\begin{aligned}
n & =10, \\
-x_{1} & =x_{10}=.9210680558, \\
-x_{2} & =x_{9}=.6318113569, \\
-x_{3} & =x_{8}=.5819174110 \\
-x_{4} & =x_{7}=.3836617341, \\
-x_{5} & =x_{6}=0.0 .
\end{aligned}
$$

The minimum of $(2)$ for $n=10$ is $0.30362 \times 10^{-6}$.

$$
\begin{aligned}
n & =11, \\
-x_{1} & =x_{11}=.9267650132, \\
-x_{2} & =x_{10}=.7049241194, \\
-x_{3} & =x_{9}=.5179229707, \\
-x_{4} & =x_{8}=.4574029197, \\
-x_{5} & =x_{6}=x_{7}=0.0 .
\end{aligned}
$$

The minimum of (2) for $n=11$ is $0.34535 \times 10^{-6}$.

It has been numerically verified that the reported numbers are local minima of (2). Also the surface corresponding to (2) of which the minimum was sought is fairly "flat" in that (2) is not very sensitive to "large" changes in the values of the nodes.

3. Generalizations. The above approach may be generalized to a minimization of the $l^{2}$-norm of the deviations of the first $k$ monomials rather than the first $n+1$ monomials, $k \geqq n+1$. The case $k=\infty$, with no constraints on the weights or nodes, has been considered by Wilf [7]. With $k=\infty$, the weight constrained to be $2 / n$, and the nodes symmetric, the following equations must be satisfied: 
(3)

$$
\begin{aligned}
\frac{4}{n} \sum_{i=1}^{[n / 2]} \frac{x_{j} x_{i}{ }^{2}}{\left(1-x_{j}{ }^{2} x_{i}{ }^{2}\right)^{2}} & =\frac{1}{x_{j}\left(1-x_{j}{ }^{2}\right)}-\frac{1}{x_{j}{ }^{2}} \tanh ^{-1} x_{j} \\
& =\int_{-1}^{+1} \frac{x_{j} x^{2}}{\left(1-x_{j}{ }^{2} x^{2}\right)^{2}} d x \quad j=1, \cdots,[n / 2],
\end{aligned}
$$

i.e. we require precision for the functions

$$
\frac{x_{j} x^{2}}{\left(1-x_{j}{ }^{2} x^{2}\right)^{2}} \quad j=1, \cdots,[n / 2] .
$$

Equation (3) cannot be derived by a specialization of Wilf's results. A similar case has been discussed by Valentin [6, p. 43]. It should also be noted that the case $k=\infty$ corresponds to a Chebyshev type of minimum norm quadrature [5, 8]. The generalized Newton's method was applied to the case $n=8$. With the initial approximation $(.9, .6,0.0,0.0)$, the following solution to (3) was obtained:

$$
\begin{aligned}
& n=8, \quad k=\infty, \\
& -x_{1}=x_{8}=.9196965709, \\
& -x_{2}=x_{7}=.6137864317, \\
& -x_{3}=x_{6}=0.0, \\
& -x_{4}=x_{5}=0.0 .
\end{aligned}
$$

Finite values of $k$ were also considered. The following table gives those results:

$\begin{array}{lllll}k & 10 & 12 & 20 \\ -x_{1}=x_{8}=.8869315744 & .8936970519 & .9056780702 \\ -x_{2}=x_{7}=.6967120629 & .6817522473 & .6510495793 \\ -x_{3}=x_{6}=.0 & .0 & .0 \\ -x_{4}=x_{5}= & .0 & .0 & .0 \\ \text { Min. } & .222382 \times 10^{-2} & .277134 \times 10^{-2} & .540009 \times 10^{-2} \\ k & 80 & 160 & 200 \\ -x_{1}=x_{8}=.9195306124 & .9196963374 & .9196965409 \\ -x_{2}=x_{7}=.6142171082 & .6137867665 & .6137862383 \\ -x_{3}=x_{6}= & .0 & .0 & .0 \\ -x_{4}=x_{5}= & .0 & .0 & .0 \\ \text { Min. } & .359082 \times 10^{-1} & .452538 \times 10^{-1} & .476983 \times 10^{-1}\end{array}$

For both finite and infinite values of $k$, numerous initial approximations with nonzero nodes were used. In each of these cases, the corresponding generalized Newton sequence diverged. It appears from Eq. (3) that the trouble arose from some of the nodes being near zero.

Department of Mathematics

University of Utah

Salt Lake City, Utah 84112

1. A. Ben-Israel, "A Newton-Raphson method for the solution of systems of equations," J. Math. Anal. Appl., v. 15, 1966, pp. 243-252. MR 34 \#5273. 
2. V. I. KRylov, Approximate Calculation of Integrals, Fizmatgiz, Moscow, 1959; English transl., Macmillan, New York, 1962. MR 22 \#2002; MR 26 \#2008.

3. A. Meir \& A. Sharma, "A variation of the Tchebicheff quadrature problem," Illinois $J$. Math., v. 11, 1967, pp. 535-546. MR 35 \#7058.

4. G. M. Nielson, Nonlinear Approximations in the $l_{2}$ Norm, M.S. Thesis, Department of Mathematics, University of Utah, Salt Lake City, Utah, 1968.

5. R. E. BARnhill, "Asymptotic properties of minimum norm and optimal quadratures," Numer. Math. (To appear.)

6. R. A. VAlentin, "The use of the hypercircle inequality in deriving a class of numerical approximation rules for analytic functions," Math. Comp., v. 22, 1968, pp. 110-117. MR 36 \#6840.

7. H. S. WILF, "Exactness conditions in numerical quadrature," Numer. Math., v. 6, 1964, pp. 315-319. MR 31 \#4178.

8. H. Yanagihara, "A new method of numerical integration of Gauss type," Bull. Fukuoka Gakugei Univ. III, v. 6, 1956, pp. 19-25. (Japanese) 